Conclusions This research showed that there was a significant positive correlation between the area of housing and TG, and there was also correlation between the occupation and FPG, between living style (living with their children or not) and WHR, between the degree of physical activities and hypertension, and doing more physical activities was associated with a lower risk of hypertension.

\title{
[gw22-e0499] RELATIONS BETWEEN THE SOCIAL ECONOMIC FACTORS AND THE RELEVANT FACTORS OF CARDIOVASCULAR DISEASE
}

Wang Shujuan', Li Wenwen', Jing Yuejuan', Gao Yuzhen², Li Yafeng' 'Shanxi Medical University, Shanxi, China; ${ }^{2}$ The Second Hospital Of Shanxi Medical University, Taiyuan, China

10.1136/heartjnl-2011-300867.269

Objectives The purpose of the present study was to investigate the associations between the social economic factors and the relative factors of cardiovascular disease.

Methods A total of 4913 residents (aged 35-75 years, mean $56.98 \pm 11.06$ years) who were from Yingze community and participated in physical examination were recruited in the study. Data collection including blood pressure (BP), fasting blood glucose (FPG), cholesterol (CHOL), triglyceride (TG), waist/hip ratio (WHR), body mass index (BMI), HDLcholesterol (HDL-C), LDL-cholesterol (LDL-C), stroke history, hyperlipidemia history, coronary heart disease history and social economic factors.

Results Bivariate correlation analysis showed that there was an increasing tendency of TG with the increasing of the area of the housing $(\beta=0.03, p=0.034)$. With the help of one-way ANOVO analysis, it showed that there was a significant correlation between the occupation and FPG, and the FPG in those who were leaders was higher than that in the common group $(6.00 \pm 1.91 \mathrm{mmol} / 1$ vs $5.79 \pm 1.55 \mathrm{mmol} / \mathrm{l} ; \mathrm{p}=0.01)$. Moreover, the association can be found between the living style (living with their children or not) and WH ratio $(p=0.021)$. Binary logistic regression analysis showed that there was a significant relation between the degree of physical activities and hypertension, and the odds ratio (95\% CI) of hypertension for the residents who had more physical activities, as compared with those who had less physical activities, was 0.745 (0.600 to 0.927, $\mathrm{p}=0.008)$. 